\title{
Embedded Trusted Computing with Authenticated Non-volatile Memory ${ }^{\star}$
}

\author{
Dries Schellekens ${ }^{1}$, Pim Tuyls ${ }^{1,2}$, and Bart Preneel ${ }^{1}$ \\ ${ }^{1}$ Katholieke Universiteit Leuven, ESAT-SCD/COSIC, Belgium \\ \{dries.schellekens, bart. preneel\}@esat. kuleuven. be \\ ${ }^{2}$ Philips Research Laboratories, Eindhoven, The Netherlands \\ pim.tuyls@philips.com
}

\begin{abstract}
Trusted computing is an emerging technology to improve the trustworthiness of computing platforms. The Trusted Computing Group has proposed specifications for a Trusted Platform Module and a Mobile Trusted Module. One of the key problems when integrating these trusted modules into an embedded system-on-chip design, is the lack of on-chip multiple-time-programmable non-volatile memory. In this paper, we describe a solution to protect the trusted module's persistent state in external memory against non-invasive attacks. We introduce a minimal cryptographic protocol to achieve an authenticated channel between the trusted module and the external non-volatile memory. A MAC algorithm has to be added to the external memory to ensure authenticity. As a case study, we discuss trusted computing on reconfigurable hardware. In order to make our solution applicable to the low-end FPGA series which has no security measures on board, we present a solution that only relies on the reverse engineering complexity of the undocumented bitstream encoding and uses a physically unclonable function for one-time-programmable key storage. Clearly, this solution is also applicable to high-end series with special security measures on board. Our solution also supports field updates of the trusted module.
\end{abstract}

\section{Introduction}

Trusted computing is a promising technology to increase the security and trustworthiness of today's computing platforms. The main initiative for a new generation of computing platforms is taken by the Trusted Computing Group (TCG), a consortium of most major IT companies. The TCG defines the addition of a small hardware security module and a core initial software component, which act as roots of trust in the platform.

* The work described in this document has been partly financially supported by the IAP Programme P6/26 BCRYPT of the Belgian State (Belgian Science Policy), by the IBBT (Interdisciplinary institute for BroadBand Technology) of the Flemish Government, by the FWO project BBC G.0300.07, and in part by the European Commission through the IST Programme under Contract IST-027635 OPEN_TC. 
The initial focus of the TCG was on the open personal computer platform, resulting in the specification of a Trusted Platform Module (TPM)1. Recently, the TCG Mobile Phone Work Group (MPWG) published the specification for a Mobile Trusted Module (MTM) and proposed a reference architecture. The specification distinguishes between local and remote owner trusted modules, defines a subset of the TPM commands that have to be implemented, and describes mobile specific commands, e.g., to implement secure boot in a standardized way [1].

Currently, TPMs are typically implemented as a discrete cryptographic chip physically bound to the rest of platform (i.e., soldered to the mainboard), but chipsets with integrated TPMs are forthcoming. For the MTM specification various implementation options exist, especially because a mobile phone will contain multiple trusted modules for different stakeholders (e.g., device manufacturer and cellular operator). The MTM can be implemented in hardware as a separate dedicated chip or integrated into existing chips [2, or as software running on the main processor, possibly in a higher privileged mode 314. If the platform has to support multiple execution engines, software/virtual trusted modules can run in isolated domains provided by a microkernel or hypervisor [5]6/7.

The security of the discrete chip solution is compromised if the communication interface between the trusted module and the rest of the platform is vulnerable to attacks [8]. Additionally, the cost of an extra chip is considerable for low-end embedded devices. Hence, the integration of the trusted module into another chip is preferable. One of the main problems when embedding a security module into an existing chip or running this functionality on the application processor is the lack of on-chip Multiple-Time-Programmable (MTP) Non-Volatile Memory $(\mathrm{NVM})^{2}$. It is needed by the trusted module to securely store its persistent state, which includes cryptographic keys, authorization data and monotonic counters. Although external non-volatile memory (e.g., Flash or EEPROM) is commonly available in embedded devices, it can not store the persistent state securely. An adversary can access the communication interface to the external memory with a non-invasive hardware attack and overwrite the state with an older version, even if the state is stored in an encrypted form.

Eisenbarth et al. 10. propose the first implementation of the trusted module on reconfigurable hardware that facilitates field upgrades of the trusted module. Upgrades might be required to fix non-compliance firmware bugs [1] or to replace broken/depreciated cryptographic algorithms. They propose furthermore a new FPGA architecture with functionalities similar to trusted computing. Their construction includes a minimal root of trust, called Bitstream Trust Engine (BTE). This trust component is used to include the hardware configuration in the TCG chain of trust, by measuring the configuration bitstream before it is loaded on the FPGA. On top of this, it limits access to the trusted module's persistent state based on the loaded bitstream. Moreover their solution

\footnotetext{
${ }^{1}$ All TCG specifications referred to in this document are available at https://www.trustedcomputinggroup.org/specs/

2 MTP NVM solutions in standard CMOS logic, requiring no additional masks or processing steps, are becoming commercially available (e.g., [9]).
} 
requires partial configuration, bitstream encryption and authentication, features typically only available on high-end FPGA 3 . The BTE needs NVM inside the FPGA package to store the complete persistent state together with access control information that defines which bitstream has access to the state. In order to implement this solution, various changes have to be made to the FPGA hardware. History has shown that extensive changes to a hardware architecture are unlikely to occur and that solutions which do not need these changes are often preferred since they are more economically viable.

In this paper, we study embedded trusted computing on devices, which only have integrated One-Time-Programmable (OTP) NVM. We propose to store the trusted module's state in an external NVM chip that is extended with a security mechanism. In this way a mutual authenticated and fresh communication channel is realized between the trusted module and the external NVM. Our solution requires the addition of a MAC algorithm and some access control logic to the external NVM. We deem these changes as reasonable because some semiconductor companies already provide a similar security-enhanced NVM [12].

As a case study we look at volatile FPGAs. We propose to implement the required OTP key storage as an intrinsic Physical Unclonable Function (PUF). Intrinsic PUFs can be extracted from memory structures on the FPGA or laid down in logic [13. Our solution only relies on the complexity of full bitstream reversal and can hence be realized with current low-end SRAM FPGA technology which does not have any additional built-in security mechanisms. Bitstream reversal is defined as the reverse engineering process that transforms an encoded bitstream into the original logical design, and it is generally believed to be a hard task that takes a lot of effort [14. This difficulty is not exactly measurable and does not achieve nowadays cryptographic standards. The security of our proposal can be strengthened by bitstream protection techniques such as bitstream encryption.

This paper is organized as follows. Section 2 gives some background on classical bitstream protection techniques and PUFs. In Section 3 we describe the implementation of an integrated trusted module, propose a mechanism to protect the trusted module's persistent state and introduce an authenticated NVM scheme. Section 4 describes our solution for trusted computing on reconfigurable hardware that relies on an intrinsic PUF for key storage and allows field updates. The paper ends with a summary of our work and conclusions.

\section{Volatile FPGA Security}

Our main example for reconfigurable platforms will be SRAM based FPGAs. SRAM based FPGAs offer very large flexibility and form the bulk of the market. In this section we describe several protection technologies for SRAM based FPGAs.

\footnotetext{
${ }^{3}$ Xilinx bitstream encryption disables partial configuration, reconfiguration and readback to increase security. So the combination of partial configuration and bitstream encryption is currently unsupported.
} 


\subsection{Classical Bitstream Protection Techniques}

There are two main classical mechanisms to protect FPGA bitstreams against cloning: bitstream encryption and node locking.

Bitstream encryption provides confidentiality of the bitstream and binds it to the platform. As the name suggests, the bitstream is encrypted with a key which has to be stored in non-volatile memory on the FPGA. When the bitstream is loaded onto the FPGA, it is first fed to a decryption module. The decrypted bitstream is then used to configure the FPGA. In order to implement this technology, non-volatile memory is required on the FPGA for storage of the long-term key. This is currently only provided for the high-end FPGAs and comes at a cost since it requires different memory hardware or a battery [14].

Node locking is a technology that binds the bitstream to a specific platform. The basic idea is that the bitstream is bound to the platform by an identifier that can not be modified by an attacker and that is stored either in an external chip (e.g., Dallas Secure EEPROM [15]) or internally as a device DNA 4 or a PUF. By checking the presence of the correct identifier, the bitstream will determine whether it runs on the expected platform or not. The security of these solutions depends amongst other things on the level of obfuscation provided by the bitstream encoding (see Section 2.3).

\subsection{Physical Unclonable Function}

We briefly introduce the notion of a Physical Unclonable Function and present some examples of PUFs that are present on ICs due to process variations. Their main advantage is that they are intrinsically present on an IC, i.e., not requiring changes to the hardware. For details we refer to [13[16.

Definition 1. A PUF is a physical structure that consists of many random, uncontrollable components, that is therefore very hard to clone. The structure satisfies the following properties:

(i) It is easy to challenge the PUF and measure the response of the PUF according to the challenge. The combination of a challenge $C$ and a response $R$ is called a challenge-response pair.

(ii) A PUF has many challenge-response pairs.

(iii) Given a challenge $C$, the response $R$ of the PUF is unpredictable.

(iv) The PUF can not be reproduced by the manufacturer.

(v) The PUF should be tamper evident.

(vi) Preferably the PUF is inseparably bound to the object that it is protecting.

The main examples of PUFs that are intrinsically present in ICs in general and SRAM based FPGAs in particular, are SRAM PUFs and Silicon PUFs. SRAM PUFs were introduced in [13] and exploit the random behavior of uninitialized SRAM memory cells during startup. This behavior originates from doping variations in the transistors that make up those cells. Silicon PUFs on the other hand

\footnotetext{
${ }^{4}$ http://www.xilinx.com/products/design_resources/security/devicedna.htm
} 
were introduced in [16] and are based on the delay variations in the circuits laid down in an IC.

In order to use a PUF for key storage, the following procedure has to be followed based on a fuzzy extractor or helper data algorithm [17/18]. First, during an enrollment phase the PUF responses $R_{1}, \ldots, R_{n}$ for the challenges $C_{1}, \ldots, C_{n}$ are measured. Then for a response $R_{i}$ one generates a key $K_{i}$ and helper data $W_{i}$ using the function Gen of the fuzzy extractor. Later during the reconstruction phase, the noisy PUF response $R_{i}^{\prime}$ is measured again and by using the appropriate helper data $W_{i}$, the key $K_{i}$ is reconstructed using the procedure Rep as defined in [18.

It was shown in 13. that PUFs can be used to provide secure key storage on SRAM FPGAs without additional cost or hardware. This implies that PUFs offer a low cost solution to the IP counterfeiting problem.

\subsection{Bitstream Reversal}

Drimer defines bitstream reversal as the reverse engineering process to transform an encoded bitstream file into a logical functional description (i.e., netlist or HDL) [14]. The encoding of bitstream formats is largely undocumented and obscure. This makes full bitstream reversal practically impossible. Partial bitstream reversal, which decodes static data from bitstreams, is far easier and a tool to do so exist:5.

Hiding cryptographic keys in look-up tables and RAMs should be avoided, because they can be easily extracted. Keys derived from a PUF response are better protected against partial bitstream reversal. In order to capture the secret key, an adversary needs to recover the exact design of the PUF (i.e., type, exact location, etc.) and produce a modified bitstream with the same PUF that outputs the secret key. It is reasonable to assume that currently this is very difficult because the adversary would have to perform a full bitstream reversal, and we will take this as the main assumption in this paper.

\section{$3 \quad$ Embedded Trusted Computing}

To transform an existing platform, be it open or closed, into a TCG enabled platform, two changes are typically required.

(i) A trusted module (i.e., TPM or MTM), that acts as the root of trust for storage and reporting, needs to be added to the platform.

(ii) The first code executed on the central processor of the platform has to be immutable in order to serve as root of trust for measurement (CRTM) and/or verification.

In this section we will focus on the integration of a trusted module into an embedded system-on-chip ( $\mathrm{SoC})$ design. We will propose a scheme to store the

\footnotetext{
${ }^{5}$ The Ulogic project (http://www.uclogic.org) aims at full netlist recovery from
} closed FPGA bitstream formats, but currently it is only able to decode static data. 
trusted module's persistent state in external memory. Our solution relies on embedded one-time-programmable key storage and authenticated non-volatile memory.

\subsection{Trusted Module}

The TCG has specifications for two trusted modules: the Trusted Platform Module is primarily designed for the PC platform and the Mobile Trusted Module is more tailored for advanced mobile devices, like smartphones or PDAs. Both modules can be implemented with similar hardware, namely a microcontroller, a cryptographic coprocessor (RNG, RSA, SHA-1, HMAC), read-only memory for firmware and certificates, volatile memory and non-volatile memory. The main difference is the command set of the trusted modules, which in essence translates in a different firmware image. It is conceivable that for some embedded devices a more minimal trusted module is sufficient, but this would not fundamentally change the design of the trusted module.

The trusted module communicates with the central microprocessor of the platform over an I/O bus. The Low Pin Count bus is the standardized interface for PCs to communicate with a TPM, but for embedded systems one is free to use any system-on-chip bus (e.g., ARM's AMBA bus).

The trusted module needs volatile memory for temporary data. This includes key slots to load keys stored outside the trusted module, a number of Platform Configuration Registers that store measurements (i.e., hash values) made during startup of the platform, and information (e.g., nonces) about the concurrent authorization sessions.

\subsection{Persistent State}

Some information stored in the trusted module has to be stored persistently. We denote the persistent state of a trusted module with $\mathcal{T}$. For the TPM, it includes the Endorsement Key (EK) that uniquely identifies each TPM, the Storage Root Key (SRK) that encrypts other keys maintained by the TPM, the owner's authorization data (i.e., password), and the content of monotonic counters. The persistent state of a MTM contains similar data.

As a dictionary attack mitigation technique, the trusted module keeps track of the number of failed authorization attempts. This information should also be stored persistently. The TPM_SaveState command can be used to temporally save volatile state information (e.g., content of PCRs) in $\mathcal{T}$.

In order to sufficiently protect the persistent state $\mathcal{T}$, the following requirements have to be considered:

(i) State confidentiality: It should be infeasible to read the content of $\mathcal{T}$. Disclosure of $\mathcal{T}$ will for instance reveal the private SRK.

(ii) State integrity: Unauthorized modification of $\mathcal{T}$ should be infeasible, otherwise an adversary could for instance change the owner's password. 
(iii) State uniqueness: Cloning of the trusted module must be infeasible. Hence, copying or extraction of the EK has to be prevented.

(iv) State freshness: Replay of an old state must be infeasible. This is mainly necessary to protect the monotonicity of counters.

The TCG specifications differ regarding the last requirement. The TPM has to store its general purpose monotonic counters in physically shielded locations, i.e., tamper-resistant or tamper-evident hardware. The mobile specific monotonic counters should only be shielded from software executing outside the context of the MTM 6 . This implies that for MTMs state freshness must not be guaranteed in case of hardware attacks.

\subsection{Our State Protection Scheme}

When building embedded trusted computing platforms, it is desirable to integrate the trusted module into the application processor. This is more cost effective than a discrete chip. In addition, the overall security is improved because the communication interface between the main microprocessor and trusted module is only accessible with an invasive attack. Most of the trusted module's components are rather straightforward to integrate in a system-on-chip design. However, special attention is required to realize secure storage of $\mathcal{T}$, because the application processor typically lacks reprogrammable non-volatile memory.

We propose a protection scheme that stores the persistent state $\mathcal{T}$ that does not require MTP NVM inside the trusted module. Figure 1 gives a schematic

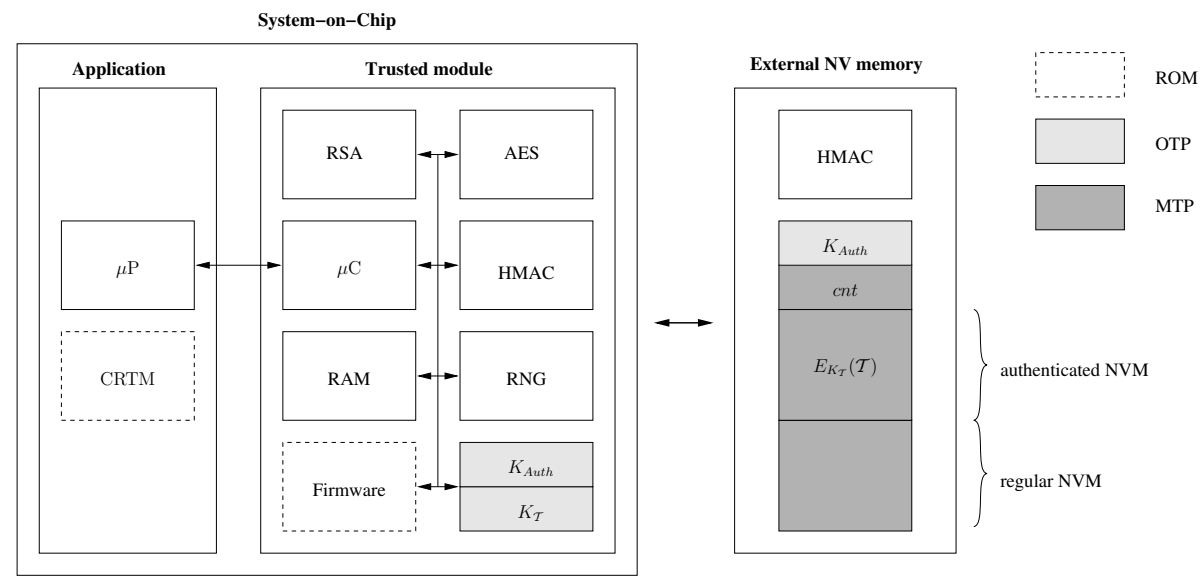

Fig. 1. Protection of the trusted module's persistent state $\mathcal{T}$ with authenticated nonvolatile memory

\footnotetext{
${ }^{6}$ The TCG MPWG intends to tighten the security requirements to the level of the TPM specifications "immediately when it becomes feasible to implement such counters in mobile phones".
} 
overview of our proposal. For efficiency reasons, our solution requires a symmetric key algorithm, more specifically AES, even though this is not part of the TCG specifications. This algorithm could also be used to protect the trusted module's key hierarchy, by making the SRK a symmetric key 7 .

The state $\mathcal{T}$ is stored externally, but encrypted: $E_{K_{\mathcal{T}}}(\mathcal{T})$. The state encryption key $K_{\mathcal{T}}$ is device specific and has to be stored in one-time-programmable NVM embedded in the trusted module. It is best to use an optimized memory encryption scheme like [19].

To protect the integrity of $\mathcal{T}$ and achieve state freshness, we propose to store the encrypted state in authenticated non-volatile memory. The communication to this security-enhanced external memory is protected by a cryptographic challenge-response protocol that uses a shared secret key $K_{\text {Auth }}$. The trusted module uses random numbers as source of freshness in the protocol, while the external memory makes use of a monotonic counter $c$. Alternatively, the external NVM can use a random number generator to generate fresh nonces.

The external memory will store the key $K_{\text {Auth }}$ and the counter $c$ in its nonvolatile memory. The OTP memory that is needed to store the keys $K_{\mathcal{T}}$ and $K_{\text {Auth }}$ inside the trusted module, can be implemented with fuses or PUFs. In Section 4.1 we will describe the realization of secure key storage with an intrinsic PUF in detail.

State Initialization. First, the keys $K_{\text {Auth }}$ and $K_{\mathcal{T}}$ have to be generated randomly and programmed in the trusted module and the external memory. In Section 3.4 we will explain how to program $K_{A u t h}$ in the external NVM and in Section 4.1 we will describe the process when a PUF is used for key storage inside the trusted module.

Next, the internal state of trusted module will be initialized. This includes the generation of the EK (in case of TPM and local owner MTM) and the SRK (in case of remote owner MTM). The device manufacturer can also create an endorsement certificate on the public EK or program the necessary information for secure boot.

\subsection{Authenticated Non-volatile Memory}

The encrypted state $E_{K_{\mathcal{T}}}(\mathcal{T})$ is stored in security-enhanced external NVM. This external memory exposes a regular memory interface and an authenticated one. Legacy read and write operations work on the regular memory range, but they will be ignored by the external NVM on the authenticated memory range. When accessing in an authenticated memory address, a cryptographic challenge-response protocol provides data origin authentication and assures a fresh communication (see Figure 2). The protocol uses a MAC algorithm keyed with a shared secret $K_{\text {Auth }}$ and nonces. The key $K_{\text {Auth }}$ is programmed in the trusted module and the external NVM during a pairing phase.

In order to implement the above protocol, the external memory contains the following components: a MAC algorithm, some control logic and internal NVM to

\footnotetext{
7 This might not be allowed because of export restrictions.
} 
Trusted module

$r \in_{R} \mathbb{Z}_{n}$

Verify MAC

Trusted module
External NV memory

$$
\begin{aligned}
& M_{i} \leftarrow \operatorname{Read}(i) \\
& c \leftarrow \operatorname{Read}(0)
\end{aligned}
$$

(a) Read protocol

\section{External NV memory}

$c \leftarrow \operatorname{Read}(0)$

Verify MAC

Write $\left(i, M_{i}^{\prime}\right)$

Write $(0, c+1)$

(b) Write protocol

Fig. 2. Cryptographic protocols to access the authenticated memory at address $i$

store the key $K_{A u t h}$ and the monotonic counter $c$. We propose to use the HMAC algorithm since this is already present in a TCG compliant trusted module. Another option is a MAC algorithm based on a block cipher, because we already added a AES coprocessor to the trusted module.

We assume that the authentication key $K_{\text {Auth }}$ is the same for the whole authenticated memory range. If more flexibility is needed, each memory address $i$ could have its own access key $K_{A u t h, i}$. For instance, Intel's authenticated Flash memory allows to specify multiple authenticated memory ranges and associates a different key with each range [12].

Read Protocol. In order to read securely from the external memory, the read command consists of the following steps (see Figure 2(a)].

1. The trusted module generates a nonce $r$.

2. The trusted module sends the address $i$ together with $r$.

3. The external memory reads the memory at address $i: M_{i}$.

4. The external memory reads the counter $c$ from the internal address 0 .

5. The external memory returns $M_{i}$ accompanied with $c$ and a MAC on $r, i$, $M_{i}$ and $c$.

6. The trusted module verifies the MAC. If the MAC is ok, it accepts the message $M_{i}$ and performs the consequent operations. Otherwise the trusted module stops all operations.

7. The trusted module store $c$ in volatile memory, because this acts as a challenge for a subsequent write operation. 
Write Protocol. In order to write data securely to the external memory, the write command consists of the following steps (see Figure 2(b)).

1. The trusted module retrieves $c$ from internal RAM (it got this value during the previous operation).

2. The trusted module sends the data $M_{i}^{\prime}$ that it wants to write at address $i$, accompanied with a MAC on $i, M_{i}^{\prime}$, and $c$.

3. The external memory reads the monotonic counter $c$ from the internal address 0 .

4. The external memory checks the MAC. If the MAC is ok, the trusted module used the same value for $c$.

5. The external memory stores the data $M_{i}^{\prime}$ address $i$ and increments the monotonic counter by writing $c+1$ to internal address 0 .

6. The external memory returns a MAC on the new counter value (i.e., $c+1$ ).

7. The trusted module verifies the MAC and determines whether the write operation was successful by verifying that the monotonic counter was incremented. If these checks fail, the trusted module stops all operations.

8. The trusted module increments the monotonic counter in volatile memory.

If $c$ gets lost, the trusted module can retrieve the current value by performing the read operation.

Pairing Phase. A trusted entity generates the authentication key $K_{\text {Auth }}$ and programs it in the trusted module and the external memory. When the different parts (trusted module, external memory) are produced by different parties, secure key distribution protocols have to be used to distribute the key safely to those parties. Typically, these protocols rely on symmetric cryptography and a key distribution center (KDC) or on asymmetric cryptography and a public key infrastructure (PKI). However, this is not the topic of this paper.

The external memory has to offer an interface that guarantees one-timeprogrammability of $K_{\text {Auth }}$. Various options to implement OTP Flash memory are described in [20].

\subsection{Security Analysis}

Conceptually our state protection scheme satisfy all the security requirements that are listed in Section 3.2. State confidentiality is provided by the symmetric encryption scheme, uniqueness by the device specific $K_{\mathcal{T}}$, and state integrity and freshness by the cryptographic protocol.

However, an adversary can also perform a denial-of-service attack, e.g., by blocking the communication to the external NVM. An attacker can also try to learn the secret keys with side channel analysis (SCA) or perform an invasive attack. When designing the cryptographic coprocessor of the trusted module and the external NVM, SCA countermeasures have to implemented.

To better protect against invasive attacks, it is advised to realize the key storage in both devices with a PUF and use an authenticated encryption scheme. 


\section{Reconfigurable Trusted Computing}

As a case study, we look firstly at volatile reconfigurable hardware. More specifically we investigate how the trusted module's secret keys are stored securely. Since volatile FPGAs do not have non-volatile memory on board, we propose to extract the secret keys from an intrinsic PUF.

The fact that the system-on-chip design is stored externally as a configuration bitstream, enables field upgrades of the trusted module. This comes however with a second security challenge to allow for secure updates. We will also describe how to protect the design against partial bitstream reversal that exposes static data. Clearly a bitstream encryption mechanism offers strengthened security.

Table 1 summarizes all data that has to be stored in the external non-volatile memory to implement our trusted module.

Table 1. Content of external non-volatile memory

\begin{tabular}{|c|c|l|}
\hline name & type & description \\
\hline$B_{S o C}$ & regular & FPGA bitstream containing SoC design \\
$W_{\mathcal{T}}$ & regular & helper data to derive state encryption key $K_{\mathcal{T}}$ \\
$W_{\text {Auth }}$ & regular & helper data to derive link authentication key $K_{\text {Auth }}$ \\
$E_{K_{\mathcal{T}}}(\mathcal{T})$ & authenticated & encrypted persistent state $\mathcal{T}$ \\
$M_{R O M}$ & authenticated & trusted module firmware ROM and CRTM \\
$P K_{R O M}$ & authenticated & public key to verify signed ROM updates \\
\hline
\end{tabular}

\subsection{Realization of Secure Key Storage}

The trusted module needs two cryptographic keys (i.e., $K_{\mathcal{T}}$ and $K_{A u t h}$ ) to access $\mathcal{T}$. These keys have to remain confidential and need to be device specific. Some low-end FPGAs, like Xilinx Spartan-3A series, have a unique device identifier, but this identifier can be read by any bitstream. A first option is to derive the keys $K_{\mathcal{T}}$ and $K_{\text {Auth }}$ from the identifier with a secret algorithm. We note however that device DNA is currently not yet present on all FPGA types, thus we will not pursue this option further.

Therefore, we propose to derive the keys $K_{\mathcal{T}}$ and $K_{A u t h}$ from an intrinsic PUF. As explained in Section 2.2, a delay PUF can be implemented with the CLBs of the FPGA. Alternatively to implement an SRAM PUF, the startup values of an uninitialized block RAM can be used. The keys will be extracted from two PUF responses $R_{\mathcal{T}}$ and $R_{\text {Auth }}$ at the point in time when needed, using the Rep function of the fuzzy extractor and corresponding helper data $W_{\mathcal{T}}$ and $W_{\text {Auth }}$ respectively. These helper data are stored in the external memory. The corresponding challenges, $C_{\mathcal{T}}$ and $C_{A u t h}$, are embedded in the FPGA's bitstream or stored in regular external NVM memory. The PUF guarantees that given the challenges its responses are still unpredictable.

Enrollment Phase. In order to use a PUF to generate keys, an enrollment phase has to be carried out. During this phase the PUF is challenged for the 
first time with the challenges $C_{\mathcal{T}}$ and $C_{A u t h}$ and the responses $R_{\mathcal{T}}$ and $R_{A u t h}$ are measured. The Gen function of the fuzzy extractor is used to generate the keys $K_{\mathcal{T}}$ and $K_{\text {Auth }}$ for the first time together with their accompanying helper data $W_{\mathcal{T}}$ and $W_{\text {Auth }}$. The helper data are then stored in the external memory. We note that this phase can be carried out during the pairing phase discussed in Section 3.4.

The system designer can choose to create a separate enrollment bitstream $B_{P U F}$ that contains the same PUF as the bitstream $B_{S o C}$ that will be deployed afterwards.

\subsection{Protection of the Bitstream}

The bitstream $B_{S o C}$ contains the system-on-chip design and is stored in regular external NVM. The following security requirements should be considered:

(i) Design integrity: Unauthorized modification of the system-on-chip design must be impossible. More specifically the integrity of a number of components should be guaranteed: the trusted module, especially its firmware, the main processor, the CRTM code, and the communication interface between the trusted module and the CPU.

(ii) Design confidentiality: The design can contain cores whose intellectual property has to be protected. Additionally, the cryptographic keys that are embedded in the trusted module, must remain secret.

(iii) Design freshness: Reconfigurable hardware allows field upgrades of the design. It must not be possible to replay an older and insecure version of the design.

Bitstream Obfuscation. On low-end reconfigurable devices we can only rely on the reverse engineering complexity of the bitstream encoding for security purposes. According to the above mentioned literature, this gives a decent level of assurance that IP cores can not easily be reverse engineered and that directed modification of the logic is difficult.

An adversary can extract the challenge $C_{\mathcal{T}}$ and $C_{\text {Auth }}$ from the bitstream, but due to the unpredictability of the PUF responses, this knowledge is insufficient to learn any information about the keys $K_{\mathcal{T}}$ and $K_{a u t h}$. In order to be successful, he must perform a full bitstream reversal and create a malicious design with exactly the same PUF as $B_{S o C}$ that outputs the secret keys. According to the state of the art [14, this is infeasible at this point in time.

Embedded ROMs. If the CRTM code and the trusted module's firmware are embedded inside the bitstream $B_{S o C}$, partial bitstream reversal will possibly reveal the contents of these embedded ROMs and perhaps enable an adversary to create a bitstream with modified code.

The easiest way to overcome this problem is by storing the code, denoted with $M_{R O M}$, in authenticated non-volatile memory. The system-on-chip design needs to be extended with some extra logic that performs the cryptographic protocol 
to access the authenticated NVM. This logic has to have access $K_{A u t h}$, which is stored with the intrinsic PUF. As a positive side effect, the trusted module does not have to use FPGA block RAMs as ROM.

Bitstream Encryption. On high-end FPGAs bitstream encryption can be used to obtain better design confidentiality. Additionally, it is difficult to make meaningful modifications to the design if the bitstream is encrypted. Typically the plain bitstream contains linear checks (i.e., CRC), so bit flips get detected. Ideally, the bitstream should be cryptographically authenticated as well, for instance with an additional MAC algorithm or by using an authenticated encryption scheme, but no commercial FPGA hardware supports this [14].

\subsection{Field Updates}

The TCG specifications define the TPM_FieldUpgrade command, but the implementation is free. We distinguish two type of field updates: firmware updates and bitstream updates. The trusted module's firmware is stored in authenticated NVM as $M_{R O M}$ and the trusted module's hardware is stored separately in regular NVM as part of bitstream $B_{S o C}$.

Firmware Updates. Firmware updates are fairly straightforward as we can use the authenticated write operations of the external memory. The system designer has to store a public key $P K_{R O M}$ inside the trusted module. We propose to store this key in authenticated NVM to protect its integrity. The trusted module has to offer an extra command that loads a signed firmware image $\operatorname{sign}_{S K_{R O M}}\left(M_{R O M}^{\prime}\right)$. The authenticated memory scheme assures that only the trusted module can overwrite $M_{R O M}$.

In order to protect against rollback8, a version number $v_{R O M}$ needs to be included in the firmware ROM: $v_{R O M} \subset M_{R O M}$. The trusted module has to check whether the version of the new firmware is higher than its own version: $v_{R O M}^{\prime}>v_{R O M}$.

Bitstream Updates. In some situations (e.g., to replace a cryptographic coprocessor), it might be necessary to update the FPGA bitstream $B_{S o C}$. It is crucial that the new bitstream includes exactly the same PUF. Otherwise, the secret keys $K_{\text {Auth }}$ and $K_{\mathcal{T}}$ become inaccessible and consequently the trusted module can no longer write to the authenticated NVM.

Because $B_{S o C}$ is stored in regular external NVM, it can always be overwritten by an older version. A possible solution to prevent this is to lock the bitstream $B_{S o C}$ to the firmware $M_{R O M}$. Every bitstream update will then be accompanied by a firmware update. The binding mechanism has to assure that new firmware $M_{R O M}^{\prime}$ does not function correctly with the old bitstream $B_{S o C}$. For instance, some extra logic in $B_{S o C}$ checks whether an expected identifier is present in $M_{R O M}$ and halts the design if this is not the case. Like the node locking schemes described in Section 2.1. this solution relies on the difficulty of bitstream reversal.

\footnotetext{
${ }^{8}$ Version rollback could be desirable because updates can cause issues.
} 
Trust Model. In our approach the system designer, creating the FPGA bitstreams and issuing firmware updates, has to be trusted.

\section{Conclusion}

In this paper we studied the integration of a trusted module into a systemon-chip design that lacks embedded reprogrammable non-volatile memory. We proposed a scheme to store the trusted module's persistent state externally in authenticated memory. Access to this external memory is protected by a minimal cryptographic challenge-response protocol that guarantees state integrity and freshness. Our solution can be made robust against most invasive attacks. This will be addressed in forthcoming work.

We also considered the implementation of trusted computing on reconfigurable hardware. In order to maximize the applicability of our solution we only relied on the complexity and obscurity of the bitstream format and used intrinsic physical unclonable functions for key storage. We also took into account field updates on the implementation.

Our main focus was on embedded trusted computing, but the components that we propose can be used in other security applications where state information has to be protected.

\section{References}

1. Ekberg, J.E., Kylänpää, M.: Mobile Trusted Module (MTM) - an introduction (November 2007), http://research.nokia.com/files/NRCTR2007015.pdf

2. Dietrich, K.: An Integrated Architecture for Trusted Computing for Java enabled Embedded Devices. In: 2nd ACM workshop on Scalable Trusted Computing - STC 2007, pp. 2-6. ACM, New York (2007)

3. Wilson, P., Frey, A., Mihm, T., Kershaw, D., Alves, T.: Implementing Embedded Security on Dual-Virtual-CPU Systems. IEEE Design and Test of Computers 24(6), 582-591 (2007)

4. Khan, M.H., Seifert, J.P., Wheeler, D.M., Brizek, J.P.: A Platform-level TrustArchitecture for Hand-held Devices. In: ECRYPT Workshop, CRASH - CRyptographic Advances in Secure Hardware, Leuven, Belgium, p. 16 (2005)

5. Berger, S., Cáceres, R., Goldman, K.A., Perez, R., Sailer, R., van Doorn, L.: vTPM: Virtualizing the Trusted Platform Module. In: Proceedings of the 15th USENIX Security Symposium, Berkeley, CA, USA, p. 21. USENIX Association (2006)

6. Zhang, X., Acriçmez, O., Seifert, J.P.: A Trusted Mobile Phone Reference Architecture via Secure Kernel. In: 2nd ACM workshop on Scalable Trusted Computing - STC 2007, pp. 7-14. ACM, New York (2007)

7. Kasper, M.: Virtualisation of a SIM-Card using Trusted Computing. Master's thesis, Private Fernfachhochschule Darmstadt (2007)

8. Kursawe, K., Schellekens, D., Preneel, B.: Analyzing trusted platform communication. In: ECRYPT Workshop, CRASH - CRyptographic Advances in Secure Hardware, Leuven, Belgium, p. 8 (2005)

9. De Vries, A., Ma, Y.: A logical approach to NVM integration in SOC design. EDN Magazine (2) (January 2007), http://www.impinj.com/pdf/EDN_NVMinSoC.pdf 
10. Eisenbarth, T., Güneysu, T., Paar, C., Sadeghi, A.R., Schellekens, D., Wolf, M.: Reconfigurable Trusted Computing in Hardware. In: 2nd ACM workshop on Scalable Trusted Computing - STC 2007, pp. 15-20. ACM, New York (2007)

11. Sadeghi, A.R., Selhorst, M., Stüble, C., Wachsmann, C., Winandy, M.: TCG inside? A Note on TPM Specification Compliance. In: 1st ACM workshop on Scalable Trusted Computing - STC 2006, pp. 47-56. ACM, New York (2006)

12. Alves, T., Rudelic, J.: ARM Security Solutions and Intel Authenticated Flash (2007), http://www .arm.com/pdfs/Intel_ARM_Security_WhitePaper.pdf

13. Guajardo, J., Kumar, S.S., Schrijen, G.J., Tuyls, P.: FPGA Intrinsic PUFs and Their Use for IP Protection. In: Paillier, P., Verbauwhede, I. (eds.) CHES 2007. LNCS, vol. 4727, pp. 63-80. Springer, Heidelberg (2007)

14. Drimer, S.: Volatile FPGA design security - a survey (December 2007), http://www.cl.cam.ac.uk/ sd410/papers/fpga_security.pdf

15. Baetoniu, C., Sheth, S.: FPGA IFF Copy Protection Using Dallas Semiconductor/Maxim DS2432 Secure EEPROMs (August 2005), http://www.xilinx.com/ support/documentation/application_notes/xapp780.pdf

16. Gassend, B., Clarke, D.E., van Dijk, M., Devadas, S.: Silicon Physical Unknown Functions. In: Atluri, V. (ed.) ACM Conference on Computer and Communications Security - CCS 2002, pp. 148-160. ACM, New York (2002)

17. Linnartz, J.P.M.G., Tuyls, P.: New Shielding Functions to Enhance Privacy and Prevent Misuse of Biometric Templates. In: Kittler, J., Nixon, M.S. (eds.) AVBPA 2003. LNCS, vol. 2688, pp. 393-402. Springer, Heidelberg (2003)

18. Dodis, Y., Reyzin, L., Smith, A.: Fuzzy Extractors: How to Generate Strong Keys from Biometrics and Other Noisy Data. In: Cachin, C., Camenisch, J.L. (eds.) EUROCRYPT 2004. LNCS, vol. 3027, pp. 523-540. Springer, Heidelberg (2004)

19. Suh, G.E., Clarke, D.E., Gassend, B., van Dijk, M., Devadas, S.: Efficient Memory Integrity Verification and Encryption for Secure Processors. In: 36th Annual International Symposium on Microarchitecture, pp. 339-350. ACM/IEEE (2003)

20. Handschuh, H., Trichina, E.: Securing Flash Technology. In: Breveglieri, L., Gueron, S., Koren, I., Naccache, D., Seifert, J.P. (eds.) 4th International Workshop on Fault Diagnosis and Tolerance in Cryptography - FDTC 2007, pp. 3-17. IEEE Computer Society, Los Alamitos (2007) 